\title{
New Prediction of the Number of Charging Electric Vehicles Using Transformation Matrix and Monte-Carlo Method
}

\author{
Hyo-Sang Go*, Joon-Hyoung Ryu**, Jae-won Kim**, Gil-Dong Kim** and Chul-Hwan Kim ${ }^{\dagger}$
}

\begin{abstract}
An Electric Vehicle (EV) is operated with the electric energy of a battery in place of conventional fossil fuels. Thus, a suitable charging infrastructure must be provided to expand the use of electric vehicles. Because the battery of an EV must be charged to operate the EV, expanding the number of EVs will have a significant influence on the power supply and demand. Therefore, to maintain the balance of power supply and demand, it is important to be able to predict the numbers of charging EVs and monitor the events that occur in the distribution system. In this paper, we predict the hourly charging rate of electric vehicles using transformation matrix, which can describe all behaviors such as resting, charging, and driving of the EVs. Simulation with transformation matrix in a specific region provides statistical results using the Monte-Carlo Method.
\end{abstract}

Keywords: Monte-Carlo method, Transformation matrix, Electric Vehicle(EV), Distribution system, Power supply and demand, Charging infrastructure, Prediction technique, Charging rate, Traffic volume, EV charging cost

\section{Introduction}

In recent times, the energy industry places priority on competition to achieve industry leaser effect in the competition among countries and companies. The indiscriminate consumption of fossil fuel for the development of industry has resulted in an increase in environmental pollution and the depletion of natural resources. The entire world has an interest in energy security and thus the need for alternative energy has gradually increased. Various industries such as alternative energy and new energy technology have been promoted in order to get rid of the dependency on fossil energy. Accordingly, public interest in electric vehicles such as Electric Vehicles (EVs) and Plug-in Hybrid Electric Vehicles (PHEVs) has been increasing with the concern for low-carbon green growth, global environmental problems, and fossil fuel shortages [1].

The Republic of Korea is currently planning to convert $10 \%$ of fossil fuel vehicles into EVs by the year 2020[2]. If unprecedented numbers of EVs are connected to the local power systems, a deterioration of the power quality or other system issues, such as overload could result. Thus, to stabilize power supply and demand, the Ministry of Trade, Industry \& Energy (MOTIE) has announced a basic plan for power supply and demand called the power-

$\doteqdot \quad$ Corresponding Author: College of Information and Communication Engineering, Sungkyunkwan University, Korea. (hmwkim@hanmail.net)

* College of Information and Communication Engineering, Sungkyunkwan University, Korea. (ghs015@hanmail.net)

** KRRI(Korea Railroad Research Institute), Korea ( \{jhryu, youngkjw, gdkim\}@krri.re.kr)

Received: March 23, 2016; Accepted: August 11, 2016 demand forecast and management plan [3]. It is essential to be able to predict the power supply and demand when establishing a power-demand management plan [4-7]. To accurately predict the power supply and demand, it is necessary to predict the number of charging EVs, connected to the distribution system. However, prediction methods do not exist in the current research regarding the forecasted charging rate of EVs. When predicting the number chargeable EVs, conditions regarding the efficiency between the maximum driving time and charging time should be considered. However, currently, the method for predicting the number of chargeable EVs is based on a simpler method, in which traffics and battery power are used, as described [3-7]. Also, Gauss method, moving average method, exponential smoothing method, and regression analysis method [8-12] are used for the prediction. These methods are based only on the past data when predicting future. Therefore, accuracy of the values obtained by the above methods cannot be secured, since not many EVs are distributed yet. Thus, in this paper, a new prediction method was used. This paper suggests a prediction method that uses currently available data such as government policies, real-time traffic, and charging cost rather than historical data to forecast future demand. Therefore, this paper suggests a prediction method that uses currently available data such as government policies, real-time traffic, and charging cost rather than historical data to forecast future demand. In Section 2, prediction techniques based on Transformation Matrix Design Conditions and determination of charged/driven EV rates with matrix and Monte-Carlo Method are discussed. In Section 3, the predicted number of charging EVs using an EV charge-rate prediction algorithm and the simulation 
results are analyzed. Finally, conclusions derived from our work are discussed in Section 4.

\section{Prediction Techniques for the Number of Charging Electric Vehicles}

\subsection{Transformation Matrix Condition}

This paper uses charging conditions[1-3, 13-16] suitable for the Republic of Korea(average real-time traffic volumes, plans to distribute electric vehicles, status of local vehicles, power consumption of EVs, and charging time) to divide the design conditions into six distinct groups to forecast the charging rate of EVs.

1) The total number of electric vehicles: The Republic of Korea has a plan to supply EVs and will implement strategies that will be established by 2020 . It has set the distribution of EVs at approximately one million, which is $10 \%$ of the production goal for compact and intermediate vehicles until 2020. After examining the number of registered compact and intermediate vehicles in the distribution system, we predicted the number of electric vehicles using the predicted number of registered compact and intermediate vehicles registered in reference [2]. X S/S refers to "X Substation" and Z D/L refers to "Z Distribution Line". X S/S and Z D/L are the actual terms used in the Korea Electric Power Corporation (KEPCO) distribution line.

$\Rightarrow$ Electric vehicle of the X S/S Z D/L: 896 units

2) Charging and Operation time: This paper assumes EV statuses of driving, resting, and charging on an hourly rate. Therefore, because EVs are assumed to charge by the hour, there are 24 charging periods in a day.

3) Battery Capacity: Vehicles in the Republic of Korea that operate on fossil fuels are segregated according to the size of the displacement (CC: Cubic Centimeter) [13]. There is no standard for the classification of EVs. Hence, in this paper, the EVs are classified similar to fossil fuel vehicles.

Table 1 presents a comparison of the capacities of EVs and fossil fuel vehicles [1]. Only compact and midsize vehicles are separated because the Republic of Korea government set the diffusion of EVs as $10 \%$ of the production goal for compact and intermediate vehicles.

Table 1. Comparison of capacity of fossil fuel vehicles and EVs

\begin{tabular}{c|c|c}
\hline Vehicle Model & Fosicle Type & EV \\
\hline Kia Motors "Ray" & Less than $1000 \mathrm{cc}$ & $16.4 \mathrm{kWh}$ \\
\hline $\begin{array}{c}\text { Renault Samsung Motors } \\
\text { "SM3" }\end{array}$ & Less than $2000 \mathrm{cc}$ & $24.4 \mathrm{kWh}$ \\
\hline
\end{tabular}

Thus, the battery capacity of EVs is set to $24.4 \mathrm{kWh}$ in this paper.

4) Charge Efficiency: This paper sets the EV's daily mileage as $40 \mathrm{~km}$, which is a fossil fuel vehicle's daily mileage according to reference [2]. Using the Calculation Method of Charging Efficiency below, it is possible to calculate the daily EV battery usage, which is $6.2 \mathrm{kWh}$. It can be fully charged in approximately two hours when the rated capacity of the home charger is set at $3.3 \mathrm{~kW}$. The Calculation Method of Charging Efficiency is used to predict the average power consumption of electric vehicles. Therefore, the maximum usable time for an EV is approximately four hours according to the calculation using the power consumption of electricity [1]. Therefore, the charging efficiency, which is calculated as the available driving time divided by the charging time, is assumed to be $50 \%$ (e.g. one hour charge $\rightarrow 30$ minutes usage).

[Calculation Method of Charging Efficiency]

- Daily average mileage of EV (a): 40km

- Fuel Efficiency(b) $: 6.5 \mathrm{~km} / \mathrm{kWh}$

- Power consumption of EV (c)=(a)/(b)): $6.2 \mathrm{kWh}$

- Charger capacity(d): $3.3 \mathrm{~kW}$

- Charging efficiency (e)=(d)/C $\times 100) \approx 50 \%$

5) Average traffic volume according to time in each season: The Republic of Korea has four distinct seasons; hence, there is a requirement to assess the power quality by season. To set the traffic volume, this paper researched the domestic traffic volume in each season and sets the seasonal number of charging EVs. Equations (1) and (2) below set the number of total EVs in each season using the domestic seasonal average traffic volumes [14] illustrated in Table 2 .

$$
\begin{aligned}
& V_{p}=\frac{V_{m}}{V_{T}} \times 100 \\
& E_{m}=\frac{V_{P}}{100} \times E_{T}
\end{aligned}
$$

where, $V_{T}$ : Total number of fossil fuel vehicles

$V_{m}$ : Seasonal number of fossil fuel vehicles

$V_{P}$ : Seasonal fossil fuel vehicle rate

$E_{T}$ : Total number of electric vehicles

$E_{m}$ : Seasonal number of electric vehicles

The hourly average traffic volume rate in Table 3 is necessary to set the average EV traffic volume in each hour [15]. By applying this data in Eqs. (3) and (4), it is possible

Table 2. Average domestic traffic volume by season

\begin{tabular}{c|c|c|c|c|c}
\hline Season & $\begin{array}{c}\text { Number of fossil } \\
\text { fuel vehicle }\end{array}$ & $\begin{array}{c}\text { Rate } \\
(\%)\end{array}$ & Season & $\begin{array}{c}\text { Number of fossil } \\
\text { fuel vehicle }\end{array}$ & $\begin{array}{c}\text { Rate } \\
(\%)\end{array}$ \\
\hline Spring & 16,183 & 95.1 & Summer & 17,022 & 100 \\
\hline Fall & 16,656 & 97.9 & Winter & 14,707 & 86.4 \\
\hline
\end{tabular}


to set the number of EVs and predict the number of charging EVs. Table 3 presents the hourly number of driving fossil fuel vehicle from real-time traffic volume, which was extracted from the distribution system data provided by reference [15]. Therefore, the above explanation was used to set the seasonal number of operating EVs.

$$
\begin{gathered}
V_{h p}=\frac{V_{h}}{V_{d T}} \times 100 \\
E_{h}=\frac{V_{h P}}{100} \times E_{m}
\end{gathered}
$$

where, $V_{d T}$ : Daily total number of fossil fuel vehicles

$V_{h}$ : Hourly average number of fossil fuel vehicles

$V_{h P}$ : Average hourly fossil fuel vehicle rate

$E_{h}:$ Hourly number of electric vehicles

$E_{m}$ : Seasonal number of electric vehicles

6) EV charging cost: KEPCO sets the load time and EV charging cost according to the hourly load. Tables 4 and 5 indicate the charging fee according to the seasonal hourly load set by KEPCO [16].

To apply the charging cost, this paper assumes that only some vehicles among the non-operating EVs that were

Table 3. Average domestic traffic volume by hour

\begin{tabular}{c|c|c|c|c|c}
\hline Time & $\begin{array}{c}\text { fossil fuel } \\
\text { vehicle }\end{array}$ & Rate(\%) & Time & $\begin{array}{c}\text { fossil fuel } \\
\text { vehicle }\end{array}$ & Rate(\%) \\
\hline 1 & 912 & 2.78 & 13 & 1542 & 4.71 \\
\hline 2 & 681 & 2.08 & 14 & 1658 & 5.07 \\
\hline 3 & 503 & 1.54 & 15 & 1713 & 5.24 \\
\hline 4 & 347 & 1.06 & 16 & 1745 & 5.33 \\
\hline 5 & 310 & 0.95 & 17 & 1707 & 5.22 \\
\hline 6 & 472 & 1.44 & 18 & 1778 & 5.44 \\
\hline 7 & 908 & 2.78 & 19 & 2070 & 6.33 \\
\hline 8 & 1717 & 5.25 & 20 & 1827 & 5.59 \\
\hline 9 & 1974 & 6.03 & 21 & 1571 & 4.8 \\
\hline 10 & 1821 & 5.57 & 22 & 1608 & 4.92 \\
\hline 11 & 1634 & 5 & 23 & 1503 & 4.6 \\
\hline 12 & 1549 & 4.74 & 24 & 1155 & 3.53 \\
\hline
\end{tabular}

Table 4. Charging fee for electric vehicle

[KWR]

\begin{tabular}{c|c|c|c}
\hline & Summer & Spring $\cdot$ Fall & Winter \\
\hline Light Load & 57.6 & 58.7 & 80.7 \\
\hline Middle Load & 145.3 & 70.5 & 128.2 \\
\hline Peak Load & 232.5 & 75.4 & 190.8 \\
\hline
\end{tabular}

Table 5. Classification of load time zone

\begin{tabular}{c|c|c|c}
\hline & Summer & Spring Fall & Winter \\
\hline Light Load & $23: 00 \sim 09: 00 \mathrm{H}$ & $23: 00 \sim 09: 00 \mathrm{H}$ & $23: 00 \sim 09: 00 \mathrm{H}$ \\
\hline \multirow{2}{*}{ Middle } & $09: 00 \sim 10: 00 \mathrm{H}$ & $09: 00 \sim 10: 00 \mathrm{H}$ & $09: 00 \sim 10: 00 \mathrm{H}$ \\
Load & $12: 00 \sim 13: 00 \mathrm{H}$ & $12: 00 \sim 13: 00 \mathrm{H}$ & $12: 00 \sim 17: 00 \mathrm{H}$ \\
& $17: 00 \sim 23: 00 \mathrm{H}$ & $17: 00 \sim 23: 00 \mathrm{H}$ & $20: 00 \sim 22: 00 \mathrm{H}$ \\
\hline & $10: 00 \sim 12: 00 \mathrm{H}$ & $10: 00 \sim 12: 00 \mathrm{H}$ & $10: 00 \sim 12: 00 \mathrm{H}$ \\
Peak Load & $13: 00 \sim 17: 00 \mathrm{H}$ & $13: 00 \sim 17: 00 \mathrm{H}$ & $17: 00 \sim 20: 00 \mathrm{H}$ \\
& & & $22: 00 \sim 23: 00 \mathrm{H}$ \\
\hline
\end{tabular}

used for more than 3 hours 30 minutes start charging during the High cost hours $(\mathrm{H})$ and some vehicles among the non-operating EVs that were used for more than 2 hours 30 minutes start charging during the Medium cost hours (M). During the Low cost hours (L), all EVs that operated for more than 30 minutes are assumed to start charging.

\subsection{Determination of charged/driven EV rate with matrix}

The total number of EVs in a specific region is fixed in a day. This means that internal behavior determines the amount of loads in a specific region. In Section II, the actual data from reference [2-3, 13-17] can be substituted for the number of driving EVs in each hour. However, the load is dependent on the number of charging EVs. Therefore, we must devise a method to determine the charging EVs from the driving EVs. For this task, we use the Monte-Carlo Method for un-determined variables in a transformation matrix. We define a matrix; $\mathrm{A}(\tau)$; that describes the rate of the number of driving EVs in a specific region at time. The components of the matrix, $\mathrm{A}_{\mathrm{i}}(\tau) \mathrm{s}$ show the status of usage in a 30 minutes that is great common divisor of charging and spend efficiencies. So, the number of component is $2 \mathrm{U}_{\mathrm{h}+1}$ where, $\mathrm{U}_{\mathrm{h}}$ is the maximum driving time. Therefore, the matrix is written as:

$$
A(\tau)=\left\{A_{1}(\tau), A_{2}(\tau), \cdots, A_{2 U_{h}+1}(\tau)\right\}
$$

Then, we define an operator which predicts next hour. This operator is $\left(2 \mathrm{U}_{\mathrm{h}+1}\right) \times\left(2 \mathrm{U}_{\mathrm{h}+1}\right)$ matrix to satisfy a dimensions of each $\tau$. We define the operator $\mathrm{B}$ will be called transformation matrix as:

$$
B: A(\tau) \rightarrow A(\tau+1)
$$

Therefore, the full form of the evolution calculation is:

$$
A_{i}(\tau+1)=\sum_{i, j=1}^{2 U_{h}+1} B_{i j} A_{i}(\tau)
$$

In Eq. (7), the indices are the time of driving, which can be determined by (i-1)/2. The transformation matrix $B$ makes $i$ hours of use at time $\tau+1$ from $j$ hours of use at time $\tau$ by the sum of the all components $\mathrm{j}$.

In a specific region, the number of EVs is fixed and matrix A describes the EVs' distribution with driving time. Therefore, the sum of all the component of rate is:

$$
\sum_{i=1}^{2 U_{h}+1} A(\tau)=1
$$

The indices of transformation matrix $\mathrm{B}$ relate to the 
behaviors of the EVs. EV behavior capacities are driving, charging, and resting. This means that the sum over $i$ at fixed $\mathrm{j}$ is one.

$$
\sum_{i, j=1}^{2 U_{h}+1} B_{i j}=1
$$

The actual data from reference $[2-3,13-17]$ is calculated to rate $\mathrm{V}(\tau)$. When a user drives i hours during an hour, this EV will be $i+2$ hours of EV use an hour later. Therefore, the relation of the actual traffic volume data and matrix form is:

$$
\sum_{i=1}^{2 U_{h}+1} B_{(i+2) i} A_{i}(\tau)=V(\tau)
$$

To be more specific, the transformation matrix has zero values that relate to impossible behaviors of the EVs during an hour. Possible behaviors are only rest, driving, and charging and possible behaviors are $\mathrm{i}, \mathrm{i}+2$, and $\mathrm{i}-1$, respectively, in transformation matrix $B$ indices. Therefore, the transformation matrix $\mathrm{B}$ is written as:

$$
\mathrm{B}=\left[\begin{array}{cccccc}
\mathrm{B}_{11} & \mathrm{~B}_{12} & 0 & \cdots & 0 & 0 \\
0 & \mathrm{~B}_{22} & \mathrm{~B}_{23} & \ddots & 0 & 0 \\
\mathrm{~B}_{31} & 0 & \mathrm{~B}_{33} & \ddots & 0 & 0 \\
0 & \mathrm{~B}_{42} & 0 & \ddots & 0 & 0 \\
\vdots & \ddots & \ddots & \ddots & \ddots & \vdots \\
0 & 0 & 0 & \cdots & \mathrm{B}_{\left(2 \mathrm{U}_{h}\right)\left(2 \mathrm{U}_{\mathrm{h}}\right)} & \mathrm{B}_{\left(2 \mathrm{U}_{\mathrm{h}}\right)\left(2 \mathrm{U}_{\mathrm{h}}+1\right)} \\
0 & 0 & 0 & \cdots & 0 & \mathrm{~B}_{\left(2 \mathrm{U}_{\mathrm{h}}+1\right)\left(2 \mathrm{U}_{\mathrm{h}}+1\right)}
\end{array}\right]
$$

From matrix B, resting, charging and using EVs are determined as $\mathrm{B}_{\mathrm{ii}}, \mathrm{B}_{(\mathrm{i}-1) \mathrm{i}}$ and $\mathrm{B}_{(1+2) \mathrm{i}}$ respectively. Fig. 1 indicates a subdivision of the transformation matrix. According to behaviors of the EVs, it can be divided into three kinds, as shown in Fig. 1.

- Upper triangular matrix is charging rate.

- Lower triangular matrix is resting rate.

- Diagonal matrix is rest rate.

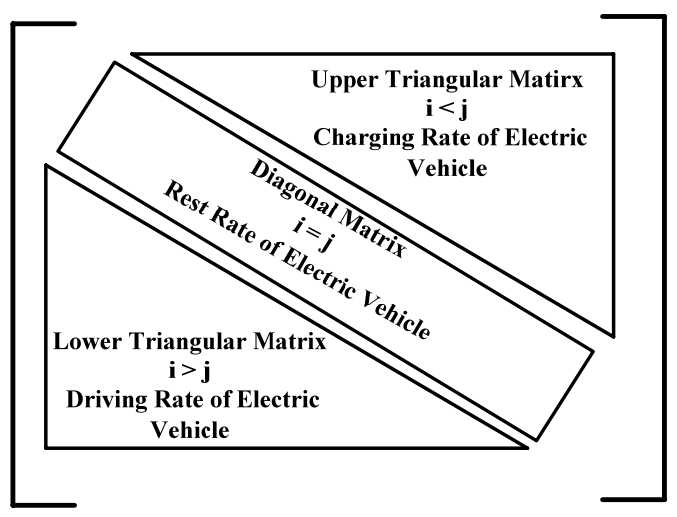

Fig. 1 Subdivision of the transformation matrix
Therefore, the numbers of each behavior are represented as:

$$
\begin{array}{ll}
\text { Rest: } & \sum_{i=1}^{2 U_{n}+1} B_{i i} A_{i}(\tau) \\
\text { Charging: } & \sum_{i=1}^{2 U_{n}+1} B_{(i-1) i} A_{i}(\tau) \\
\text { Driving: } & \sum_{i=1}^{2 U_{n}+1} B_{(i+2) i} A_{i}(\tau)
\end{array}
$$

To predict of our model, the transformation matrix B is mainly handled with a constraint the actual data (The transformation matrix $\mathrm{B}$ has to satisfy the Eq. (10)). However, there is no method to determine exact values of the components of the transformation matrix, including the driving components $\mathrm{B}_{(\mathrm{i}+2) \mathrm{i}}$.

In statistics, the Monte-Carlo Method [17] uses random numbers in a simulation with constraints and determines a solution. Using this method, it can generate random numbers in the transformation matrix B that satisfy the constraint.

\section{Calculation for the Number of Charging EVs using the Algorithm}

\subsection{EV charging rate prediction algorithm}

Within the maximum operating time range, the EV operating rate and charging rate are random. Therefore, the algorithm used to analyze the charging EV rate in this paper is essential. After computing the transformation matrix using the factors in Section II, the Monte-Carlo Method is used to calculate the charging rate because the operating and charging rates change randomly.

To minimize error, the predicted EV traffic pattern rate is realized from the quantity of real time traffic and Eq. (12); and then the error is reduced to $0.03 \%$. After calculating the operating rate, the EV charging rate is calculated with the following 10 stages.

Using the following algorithm, it is possible to predict the load increment rate, due to EVs, according to each season and time range. Fig. 2 is an algorithm that uses boundary conditions set from actual domestic data and analyzes operating electric vehicles and charging rate patterns at the same time.

\subsection{Application of the prediction algorithm in the simulation}

In case of that the maximum possible usage is four hours, the matrix A and the transformation matrix B have 9 and 81 components, respectively. The following Eq. (15) is the expression for the case: 


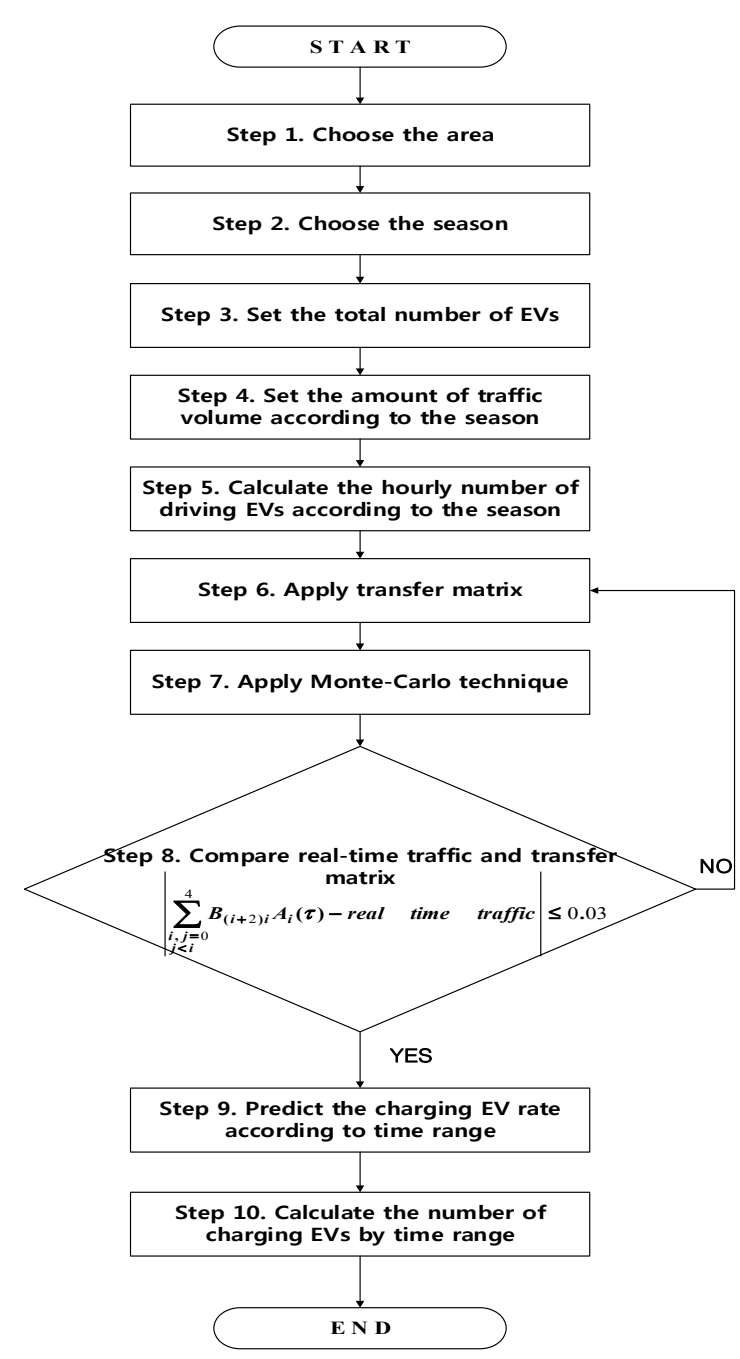

Fig. 2 Flow chart of prediction method of the number of charging electric vehicles

$\left[\begin{array}{l}\mathrm{A}_{1}(\tau+1) \\ \mathrm{A}_{2}(\tau+1) \\ \mathrm{A}_{3}(\tau+1) \\ \mathrm{A}_{4}(\tau+1) \\ \mathrm{A}_{5}(\tau+1) \\ \mathrm{A}_{6}(\tau+1) \\ \mathrm{A}_{7}(\tau+1) \\ \mathrm{A}_{8}(\tau+1) \\ \mathrm{A}_{9}(\tau+1)\end{array}\right]=\left[\begin{array}{ccccccccc}B_{11} & B_{12} & 0 & 0 & 0 & 0 & 0 & 0 & 0 \\ 0 & B_{22} & B_{23} & 0 & 0 & 0 & 0 & 0 & 0 \\ B_{31} & 0 & B_{33} & B_{34} & 0 & 0 & 0 & 0 & 0 \\ 0 & B_{42} & 0 & B_{44} & B_{45} & 0 & 0 & 0 & 0 \\ 0 & 0 & B_{53} & 0 & B_{55} & B_{56} & 0 & 0 & 0 \\ 0 & 0 & 0 & B_{64} & 0 & B_{66} & B_{67} & 0 & 0 \\ 0 & 0 & 0 & 0 & B_{75} & 0 & B_{77} & B_{78} & 0 \\ 0 & 0 & 0 & 0 & 0 & B_{86} & 0 & B_{88} & B_{89} \\ 0 & 0 & 0 & 0 & 0 & 0 & B_{97} & 0 & B_{99}\end{array}\right]\left[\begin{array}{l}\mathrm{A}_{1}(\tau) \\ \mathrm{A}_{2}(\tau) \\ \mathrm{A}_{3}(\tau) \\ \mathrm{A}_{4}(\tau) \\ \mathrm{A}_{5}(\tau) \\ \mathrm{A}_{6}(\tau) \\ \mathrm{A}_{7}(\tau) \\ \mathrm{A}_{8}(\tau) \\ \mathrm{A}_{9}(\tau)\end{array}\right]$

In the transformation matrix $\mathrm{B}$, there are 24 values which can have non-zero value. The degree of freedom of the 24 components is 15 because of the relation of the equations (9) and (10). In our model, we construct model constraints with dependences on the electricity cost. We set the behaviors as:

- Low electricity cost: All EVs that have been used are charging.

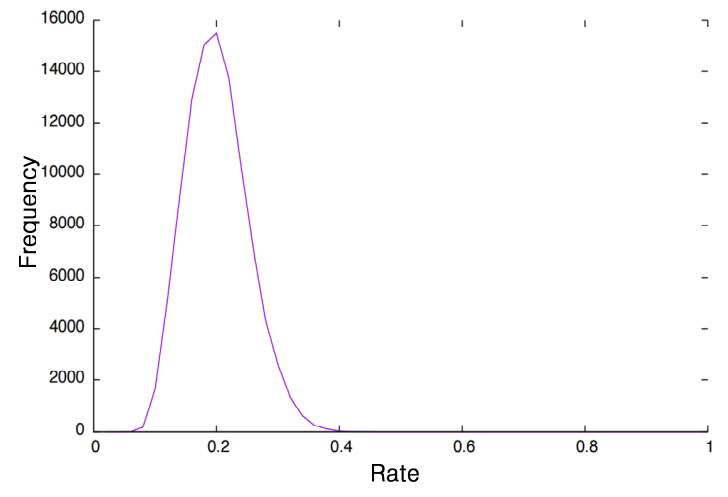

Fig. 3 Distribution chart of Hourly number of charging EVs at 04AM

- Medium electricity cost: EVs used more than 2.5 hours are charging.

- High electricity cost: EVs used more than 3.5 hour are charging.

The 15 components are undetermined by constraints. Therefore, simulation with these 15 components is computed using the Monte-Carlo Method. To generate the random numbers, we use the following procedure. We set the traffic volume to determine 15 components. The 7 components of matrix B that relate to the traffic volume Eq. (10) require 6 random numbers to satisfy the traffic volume because the sum of the 7 components is equal to the traffic volume. We generate 6 random numbers first, then generate the others to satisfy Eq. (9). Eq. (9) can generate 9 equations and each equation is the sum of two or three components. Numbers previously determined in the first step, however, reduce the degree of freedom in each Eq. (9). Therefore, we generate only 8 additional random numbers to simulate our model. For computation, we use Intel FORTRAN77.

Moreover, in order to apply different electricity price by different hours, the demand for charging is randomly selected, and, when the driving time is long, charging is conducted automatically and regardless to the price, by the algorithm. In other words, an electric vehicle which was driven for over 3 hours and 30 minutes is expected to be recharged, by its essential need for more electricity. Predictions, satisfying the above assumption and demands for charging, were numerically calculated. To find a more precise initial value at the hour or 01 , the predictions reflected a 48-hour based timeline. Moreover, in order to increase accuracy furthermore, a Monte-Carlo simulation was conducted, for 100 thousand times. Fig. 6 indicates, as a result of the simulation regarding the charging rate of the hour 04 , that approximately $20 \%$ of EVs are charging with the highest frequency.

\subsection{Prediction results of charging electric vehicles according to time range in each season}

Fig. 4 indicates the EV charging rate according to the 
Table 6. Hourly number of electric vehicles using transformation matrix and monte-carlo method

\begin{tabular}{c|c|c|c|c|c|c|c|c|c}
\hline Time & Spring & Summer & Fall & Winter & Time & Spring & Summer & Fall & Winter \\
\hline 1 & 461 & 485 & 475 & 421 & 13 & 214 & 226 & 221 & 205 \\
\hline 2 & 358 & 376 & 368 & 326 & 14 & 227 & 239 & 234 & 214 \\
\hline 3 & 239 & 251 & 246 & 225 & 15 & 242 & 254 & 249 & 228 \\
\hline 4 & 163 & 171 & 168 & 152 & 16 & 256 & 270 & 264 & 242 \\
\hline 5 & 109 & 115 & 112 & 100 & 17 & 289 & 304 & 298 & 246 \\
\hline 6 & 73 & 77 & 75 & 67 & 18 & 290 & 305 & 298 & 252 \\
\hline 7 & 63 & 66 & 65 & 57 & 19 & 285 & 299 & 293 & 247 \\
\hline 8 & 83 & 87 & 85 & 75 & 20 & 303 & 319 & 312 & 281 \\
\hline 9 & 80 & 84 & 82 & 73 & 21 & 324 & 341 & 333 & 295 \\
\hline 10 & 128 & 134 & 132 & 112 & 22 & 319 & 336 & 329 & 276 \\
\hline 11 & 163 & 171 & 167 & 148 & 23 & 608 & 639 & 626 & 550 \\
\hline 12 & 204 & 215 & 210 & 187 & 24 & 571 & 601 & 588 & 518 \\
\hline
\end{tabular}

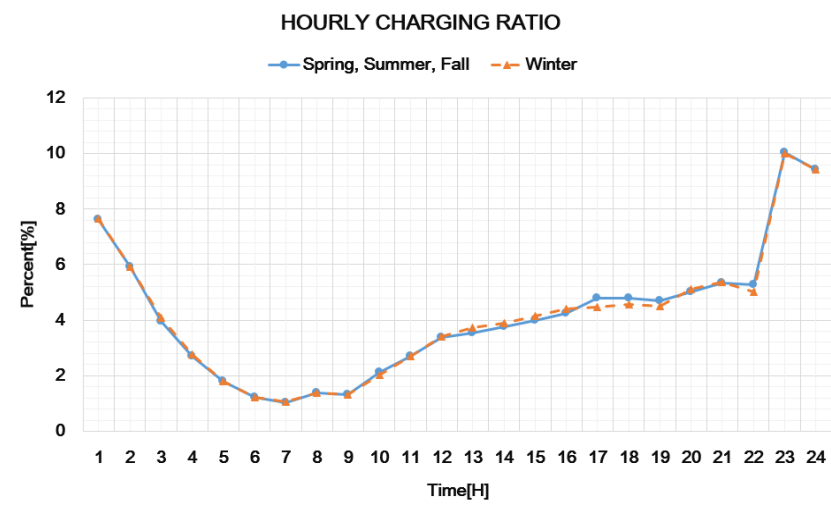

Fig. 4 Hourly charging rate curve according to seasons

time range. It uses actual domestic data (Republic of Korea's real-time amount of traffic and electric charging cost) and the prediction method elaborated in Section III. The maximum hourly charging rate is one. The reason why spring, summer, and fall are the same rate is that the time when charging costs change is the same for these seasons. Further, the charging rate changes according to the traffic rate and electric charging cost. Moreover, the charging rate is similar for all seasons; however, it increases approximately $33 \sim 35 \%$ during the period beginning at 23:00 when the price decreases.

However, as indicated in Fig. 4, the charging rate is more sensitive to the charging cost than the amount of traffic. This is because the charging ratio during the time when the cost changes (between 12:00 to 20:00) is different for spring, summer, and fall. As such, the electric cost influences the demand of the users [18-19].

Table 6 presents the number of predicted electric charging vehicles according to the charging rate in Fig. 3. Although the charging rate is similar, the number of EVs differs because the EV usage per day is different according to the season. Therefore, a maximum difference of 100 EVs can be seen during the period beginning at 23:00, which is expected to be the time when the most electric vehicles are being charged.
Table 7. Compare prediction technique and actual data

\begin{tabular}{c|c|c|c}
\hline & Prediction Technique & Actual Data[16] & Error Factor \\
\hline Light Load & $45.2 \%$ & $41.4 \%$ & $3.8 \%$ \\
\hline Middle Load & $33 \%$ & $33.8 \%$ & $0.8 \%$ \\
\hline Peak Load & $21.8 \%$ & $24.8 \%$ & $3 \%$ \\
\hline
\end{tabular}

\subsection{Error rate of predicting charging electric vehicles}

To determine the accuracy of the prediction method suggested by this paper, the following is a comparison between this paper's results and the numbers from 2013 according to light load, middle load and peak load times.

The actual data is not distributed according to time and season; rather, it is distributed according to light load, middle load and peak load. Hence, this paper converts its numbers into light load, middle load and peak load as presented in Table 7 for easier comparison.

\section{Conclusion}

The prediction method suggested in this paper determines the predicted hourly charging EV rate using a transformation Matrix; and forecasts the number of operating electric vehicles according to EV charging cost, season, difference in real-time traffic volume, and possible increase of EV usage.

As indicated in the results in Section 4, the greatest number of customers who wished to charge was during the period beginning at 23:00, when the charging cost was least. Moreover, the number of charging EVs was higher in the summer by $5 \%$ compared to spring, $2.1 \%$ compared to fall, and $13.6 \%$ compared to winter. This prediction of the charging rate was largely influenced by the charging cost. This is because there are many vehicles that do not start charging owing to the high charging cost. Therefore, there is clear risk that a large number of electric vehicles may charge during the day when the charging cost is reduced. To elaborate, it is predicted that all electric vehicles could be connected to the distribution system at the same time during the period beginning at 23:00, when the charging cost is the least. As such, there is a need to mitigate such concentration during the lowest cost hours.

In this paper, the error factor was as low as $3.8 \%, 0.8 \%$, and $3 \%$ based on light, middle, and peak loads respectively. However, it is unlikely that the actual charging rate data of the $\mathrm{EV}$ is accurate in this paper, because the penetration rate of the EVs is low. Owing to these issues, we designed the prediction technique such that the predicted values reflect the actual data. An advantage of the prediction method suggested by this research is that it can be flexibly applied to actual data such as real-time traffic, battery capacity, and length of operation per day. Therefore, even if the actual charging rate data change, it is always possible to reduce the error factor through the actual data. Moreover, 
this research can be used to predict and prevent potential problems that may occur in the distribution system when many electric vehicles are connected at the same time by predicting the charging rate changes according to the increase of electric vehicle usage. Therefore, when applied to distribution automation system, it can be built very advanced distribution system. The reason is that it can be predict future power consumption of electric vehicle. We expect to lower the event(Voltage sag, Blackout and so on) occurred in the distribution system.

\section{Acknowledgements}

This research was supported by a grant from $R \& D$ Program of the Korea Railroad Research Institute, Republic of Korea.

\section{References}

[1] Hyo-Sang Go, Doo-Ung Kim, Jun-Hyeok Kim, SoonJeong Lee, Seul-Ki Kim, Eung-Sang Kim, ChulHwan Kim, "A Study on Voltage Sag Considering Real-Time Traffic Volume of Electric Vehicles in South Korea", Journal of Electrical Engineering \& Technology, Vol. 10, No. 4, pp. 1492-1501, 3. 2015.

[2] Korea Smart Institute, "A Research of Charging Infrastructure for Electric Vehicle", 2010. 9.

[3] Ministry of Trade, Industry \& Energy, "The 6th Basic Plan of Long-Term Electricity Supply and Demand", 2. 2013.

[4] Jian-chang Lu, Xingping Zhang, Wei Sun, "A Realtime Adaptive Forecasting Algorithm for Electric Power Load", Transmission and Distribution Conference and Exhibition IEEE/PES, pp. 1-5, 2005.

[5] A. Sfetsos, "Short-term load forecasting with a hybrid clustering algorithm", Generation, Transmission and Distribution IEE Proceedings, Vol. 150, issue 3, pp. 257-262, 3. 2003.

[6] V. H. Hinojosa, A. Hoese, "Short-Term Load Forecasting Using Fuzzy Inductive Reasoning and Evolutionary Algorithms", IEEE Transactions on Power Systems, Vol. 25, No. 1, pp. 565-574, 2. 2010.

[7] Yang Wang, Qing Xia, Chongqing Kang, "Secondary Forecasting Based on Deviation Analysis for ShortTerm Load Forecasting", IEEE Transactions on Power Systems, Vol. 26, No. 2, pp. 500-507, 3. 2011.

[8] Kristien Clement-Nyns, Edwin Haesen, Johan Driesen, "The Impact of Charging Plug-In Hybrid Electric Vehicles on a Residential Distribution Grid", IEEE Transactions on Power Systems, Vol. 25, No. 1, pp. 371-380,1. 2010.

[9] Sara Deilami, Amir S. Masoum, Paul S. Moses, Mohammad A. S. Masoum,"Real-Time Coordination of Plug-In Electric Vehicle Charging in Smart Grids to Minimize Power Losses and Improve Voltage Profile", IEEE Transactions on SMART GRID, Vol. 2, No. 3, pp. 456-467, 9. 2011.

[10] KejunQian, Chengke Zhou, Malcolm Allan, Yue Yuan, "Modeling of Load Demand Due to EV Battery Charging in Distribution Systems", IEEE Transactions on Power Systems, Vol. 26, No. 2, pp. 802-810, 5. 2011.

[11] Yijia Cao, Shengwei Tang, Canbing Li, Peng Zhang, Yi Tan, Zhikun Zhang, Junxiong Li, "An Optimized EV Charging Model Considering TOU Price and SOC Curve", IEEE Transactions on SMART GRID, Vol. 3, No. 1, pp. 388-393, 3. 2012.

[12] Luis PieltainFernández, Tomás Gómez San Román, Rafael Cossent, Carlos Mateo Domingo, Pablo Frías, "Assessment of the Impact of Plug-in Electric Vehicles on Distribution Networks", IEEE Transactions on Power Systems, Vol. 26, No. 1, pp. 206213, 2. 2011.

[13] Korea Ministry of Government Legislation, "MOTOR VEHICLE MANAGEMENT ACT", 8. 2013.

[14] Korea Institute of Civil Engineering and Building Technology, TMS (Traffic Monitoring System), 2012

[15] MLIT(Ministry of Land, Infrastructure and Transport), "Statistical yearbook of MLIT", 12. 2013.

[16] Korea Electric Power Corporation(KEPCO), "www.kepco.co.kr"

[17] P. Wijesinghe, U. Gunawardana, R. Liyanapathirana "Transition Matrix Monte Carlo Technique for Outage Probability Estimation in MIMO Channels", Communications Theory Workshop (AusCTW), p. 130-135, 1. 2011.

[18] Dong-Joo Kang, Sun-Joo Park, Soo-Jung Choi, Seong-Jae Han, "A Study on Design of Home Energy Management System to Induce Price Responsive Demand Response to Real Time Pricing of Smart Grid", Journal of KIIEE, Vol. 25, No. 11, pp. 39-49, 11. 2011.

[19] Hee-jung Hong, Soek-man Han, al-ho Kim, “A Study on the Change in Production Costs and Electricity Tariffs with the Introduction of Renewable Portfolio Standard", Journal of KIEE, Vol. 58, No. 4, pp. 708717, 4. 2009.

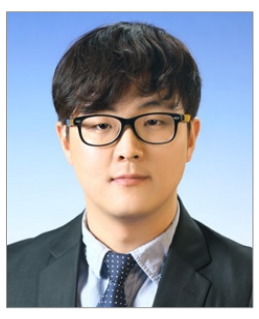

Hyo-Sang Go was born in Korea, 1985. He received his B.S in School of Department of Physics from Chungbuk National University, Korea, 2010. At present, he is working on his Master and Ph. D thesis at Sungkyunkwan University. His research interests include power system transients, protection and power quality with electric vehicles. 


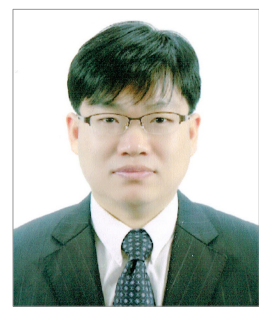

Joon-Hyoung Ryu was born in Korea, in 1972. He received his Ph.D. in Electronics engineering from Ajou University, Suwon, Korea, in 2005. At present, he is working for Korea Railroad Research Institute as a senior research engineer. His research interests include propulsion control system, power conversion systems, energy storage system, power electronics, and rolling stocks.

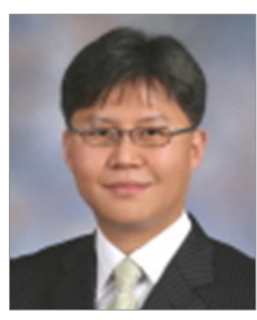

Jae-won Kim received the B.S. and M.S. degree in electrical engineering from KOREA University, Seoul, Korea, in 2006 and 2008. He is currently working toward the $\mathrm{Ph}$. D degree at the same University. He joined KRRI (Korea Railroad Research Institute), Uiwang-si, Korea, in 2011. He is presently senior researcher. His research interests include voltage stability of electric power system, analysis of railway electric power grids and reliability of railway vehicle components.

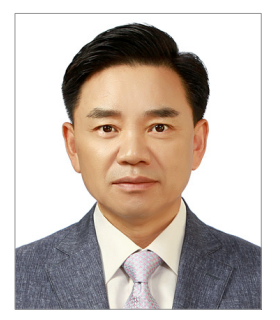

Gil-Dong Kim He received his B.S degree in Electronics engineering in 1986, M.S degree in 1991 and Ph.D from Myongji University in 2003. At present, he is working for Korea Railroad Research Institute and also Propulsion System Team as a Head/ Chief research engineer. His research interests include propulsion control system, power conversion systems, power electronics, and rolling stocks.

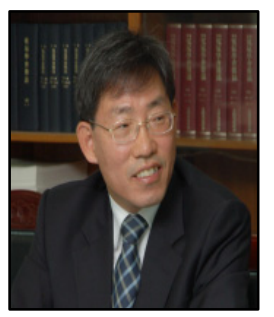

Chul-Hwan Kim was born in Korea, 1961. In 1990 he joined Cheju National University, Cheju, Korea, as a full-time Lecturer. He has been a visiting academic at the University of BATH, UK, in 1996, 1998, and 1999. Since March 1992, he has been a professor in the School of Electrical and Computer Engineering, Sungkyunkwan University, Korea. His research interests include power system protection, artificial intelligence application for protection and control, the modelling/protection of underground cable and EMTP software. He received his B.S and M.S degrees in Electrical Engineering from Sungkyunkwan University, Korea, 1982 and 1984, respectively. He received a Ph.D in Electrical Engineering from Sungkyunkwan University in 1990. Currently, he is a director of Center for Power IT (CPIT) in Sungkyunkwan University. 